\title{
Fachärzte blicken gelassener auf die Praxisabgabe als Hausärzte
}

\begin{abstract}
Die Mehrheit der niedergelassenen Ärzte blickt mit Angst auf den Verkauf ihrer Praxis, weil sie fürchten, keinen Nachfolger zu finden. Nur noch jeder vierte Arzt glaubt, dass er mehr als $100.000 €$ für seine Praxis erlösen kann. Die Bedeutung des Praxisverkaufs für die Altersvorsorge hat sich offensichtlich relativiert.
\end{abstract}

$\mathrm{M}$ ehr als 1.000 Ärzte beteiligten sich an einer Umfrage in der „Ärzte Zeitung“, im „WirtschaftsTipp“ und im Internet, die die Fachverlagsgruppe Springer Medizin und die Deutsche Apothekerund Ärztebank initiiert hatten.

Das Bild, das die Umfrage mit 700 beteiligten Hausärzten und 300 Fachärzten vermittelt, ist allerdings nicht durchweg negativ. So sehen die Fachärzten die Situation deutlich entspannter: Von ihnen halten immerhin mehr als $40 \%$ die Praxisnachfolge für gesichert oder für wahrscheinlich, und auch beim Praxiswert sind die Erwartungen der Fachärzte höher als diejenigen der Hausärzte.

Hinzu kommt, dass viele Ärzte auf die Schwierigkeiten reagieren, um so ihre

\section{Nachträglich bemerkter Abrechnungsfehler: Honorarbescheid ist trotzdem rechtens}

\begin{abstract}
Abrechnungsfehler, die durch das Personal oder die Software verursacht wurden und erst nach Ablauf der von der KV gesetzten Frist zutage treten, müssen nachträglich nicht anerkannt werden.
\end{abstract}

$\mathrm{n}$ einem Fall, der vor dem Sozialgericht

Marburg verhandelt wurde (Az.: S 12 KA 732/09), wollte eine hausärztlich tätige Internistin nachträglich Leistungen von der KV Hessen erstattet bekommen, da die Honorarabrechnung nicht vollständig gewesen war. Als Grund für die mangelhafte Abrechnung nannte sie die Unerfahrenheit ihrer Medizinischen Fachangestellten (MFA) und eine fehlerhafte Software. Wegen Arbeitsverweigerung hatte sie einer MFA am Ende der Probezeit gekündigt. Aus diesem Grunde musste sie zeitweise mit Personal zurecht kommen, das nicht eingearbeitet war.

Bemängelt hatte die Ärztin, dass die Restzahlung für das Quartal IV/2008 in abändern. individuelle Situation zu verbessern: JeAbgabe investieren, um die Praxis leichter verkaufen zu können. Ein weiteres Drittel der Umfrageteilnehmer will versuchen, über die Zusammenarbeit mit einem Juniorpartner einen Nachfolger $\mathrm{zu}$ finden. Und jeder sechste strebt an, die Praxis in eine Kooperation einzubringen.

Im Internet sind die ausführlichen Ergebnisse dieser Umfrage und auch die Antworten von Experten der Deutschen Apotheker- und Ärztebank zu den wichtigsten Fragen von Teilnehmern zur Praxisabgabe zu finden: www.aerztezeitung. de (Suchwort: Praxisabgabe)

Hauke Gerlof weils etwa ein Drittel will noch vor der

Medizinische Fachangestellte: Beliebter Beruf, aber kein hohes Ansehen

Die Zahlen können sich sehen lassen: 14.695 Medizinische Fachangestellte (MFA) starteten 2010 ihre Ausbildung in deutschen Arztpraxen. Damit liegen die MFA in der Rangliste aller Ausbildungsberufe bundesweit auf Platz acht. Das lässt sich dem Berufsbildungsbericht 2011, der vom Bundesministerium für Bildung und Forschung und dem Bundesinstitut für Berufsbildung (BiBB) veröffentlicht wird, entnehmen. Kleiner Wehrmutstropfen: 37,3\% aller Bewerber 2010 und immerhin über ein Viertel der weiblichen Bewerber - waren der Ansicht, dass der Beruf der/des MFA ihnen zu keinem guten Image bei Bekannten verhelfen wird.

Rebecca Höhl

Unverbindliche Auskünfte des Fiskus gelten nicht

Unverbindliche Auskünfte des Fiskus werden ungültig, wenn sich die Rechtslage ändert, so der Bundesfinanzhof (Az.: XI R 30/09). Eine Psychotherapeutin hatte Gutachten verfasst früher umsatzsteuerfrei. Eine entsprechende Auskunft erteilte 1997 das Finanzamt. „Eine verbindliche Auskunft kann ich Ihnen leider nicht erteilen", hieß es aber. Seit 2000 sind Gutachten aber nur von der Umsatzsteuer befreit, wenn sie der Diagnose und Heilbehandlung dienen. Dies war bei den Gutachten nicht der Fall. Die Psychotherapeutin musste nachzahlen.

Martin Wortmann

Höhe von etwa $7.000 €$ nur halb so groß wie sonst üblich ausgefallen sei. Eine Überprüfung der Abrechnung habe ergeben, dass die Ziffer 01950 für das tägliche Erscheinen eines Patienten, der mit Methadon substituiert werde, vergessen worden sei. Sie beantragte daher, die KV solle den Honorarbescheid noch einmal

Das Gericht wies die Klage der Ärztin ab. Die Qualifikation der Mitarbeiter sei ausschließlich der Ärztin zuzurechnen, so die Richter in ihrer Begründung. Sie allein habe es in der Hand, wen sie einstelle und mit welchen Aufgaben sie diese Person betraue. Auch Fehler bei der Software seien der Ärztin zuzurechnen.
Praxisverlegung gilt nicht als Neugründung

Vertragsärzte, die ihre Praxis innerhalb des Ortes verlegen, können nicht die honorarrechtlichen Vorteile einer Neugründung beanspruchen. Das geht aus einem Urteil des Landessozialgerichts Rheinland-Pfalz hervor (Az.: L 5 KA 4/10). Es wies die Klage einer HNOÄrztin ab, die sich nach einem Umzug beim Honorar benachteiligt fühlte. Nachdem sie ihre Praxis aus einer ungünstigen Nebenstraße an einen zentraleren Standort verlegt hatte, wollte sie wie eine Neupraxis wachsen können. Die KV lehnte dies ab.

Martin Wortmann 